DOI: $10.35381 /$ racji.v4i7.365

\title{
La intermediación gubernamental en la implementación de e-commerce para pequeños productores agrícolas
}

\section{The governmental intermediation in the implementation of e-commerce for small agricultural producers}

\author{
Alfredo Mauricio Astudillo Mamarandi \\ aastudillo@unach.edu.ec \\ Universidad Nacional de Chimborazo, Riobamba \\ Ecuador \\ https://orcid.org/0000-0001-5204-4927 \\ Mayra Gissela Lucio Solano \\ mlucio@ucem.com.ec \\ Unión Cementera Nacional -Tesorería, Riobamba \\ Ecuador \\ https://orcid.org/0000-0002-7330-2769
}

Recibido: 18 de marzo del 2019

Aprobado: 02 de junio del 2019

\section{RESUMEN}

Este documento asume el objetivo de realizar un estudio en el ámbito legal y económico con el fin de analizar la implementación de un e-commerce, auspiciada por una institución gubernamental. Por sus características y puntos de interés se dividió en 2 sub fases, es así que se plantearon entrevistas semiestructuradas dirigidas a 3 profesionales multidisciplinarios dentro del área legal para determinar si la institución auspiciante puede tener injerencia en este tipo de proyectos. Además, en el ámbito financiero, por su naturaleza se realizó una relación beneficio costo y se puso de manifiesto un análisis comparativo entre ser o no auspiciado por un ente del estado ecuatoriano. Todos los elementos de juicio utilizados en esta investigación promulgaron un positivismo a la implementación de un e-commerce y en el cual se evidenció el alto grado de viabilidad.

Descriptores: E-commerce; Comercio; Constitución. 
lustitia Socialis. Revista Arbitrada de Ciencias Jurídicas.

Año IV. Vol. IV. N6. Enero - Junio 2019

Hecho el depósito de Ley: FA2016000064

ISSN: 2542-3371

FUNDACIÓN KOINONIA (F.K). Santa Ana de Coro, Venezuela

Alfredo Mauricio Astudillo Mamarandl; Mayra Gissela Lucio Solano

\begin{abstract}
This document assumes the objective of conducting a study in the legal and economic field in order to analyze the implementation of an e-commerce, sponsored by a government institution. Due to its characteristics and points of interest, it was divided into 2 sub phases, which is how semi-structured interviews were directed to 3 multidisciplinary professionals within the legal area to determine if the sponsoring institution can have interference in this type of project. In addition, in the financial field, due to its nature, a cost-benefit relationship was made and a comparative analysis was revealed between being or not sponsored by an entity of the Ecuadorian state. All the elements of judgment used in this investigation promulgated a positivism to the implementation of an ecommerce and in which the high degree of viability was evidenced.
\end{abstract}

Descriptors: E-commerce; Commerce; Constitution.

\title{
INTRODUCCIÓN
}

En el Ecuador hay instituciones gubernamentales encargadas del desarrollo de estos mercados agrícolas, el Ministerio de Agricultura, Ganadería, Acuacultura y Pesca, el cual dentro de sus competencias está, "regulan, norman, facilitan, controlan y evalúan la gestión de la producción agrícola y pesquera del país" (Ministerio de Agricultura, Ganadería, Acuacultura y Pesca, 2017). El Ministerio de Agricultura, Ganadería, Acuacultura y Pesca (MAGAP) dentro de sus programas y servicios genera redes comerciales en donde "gestiona e impulsa estrategias para la comercialización de alimentos provenientes de organizaciones campesinas en circuitos cortos con la finalidad de incentivar relaciones comerciales, más equitativas para el pequeño y mediano productor." (MAGAP, 2017), este programa es replicado en las 9 zonas administrativas que tiene el estado ecuatoriano.

El entorno socioeconómico, tecnológico y los cambios del enfoque del marketing para ventas, hacen evolucionar a los consumidores, ya que pone al alcance de toda persona el producto o servicio más común como complejo, dentro del mercado virtual. Por ejemplo: alimento, prendas de vestir, bebidas de refresco, así como maquinaria pesada, o incluso bienes intangibles está a un clic del consumidor final, si a mercados digitales 
lustitia Socialis. Revista Arbitrada de Ciencias Jurídicas.

Año IV. Vol. IV. N6. Enero - Junio 2019

Hecho el depósito de Ley: FA2016000064

ISSN: 2542-3371

FUNDACIÓN KOINONIA (F.K). Santa Ana de Coro, Venezuela

Alfredo Mauricio Astudillo Mamarandl; Mayra Gissela Lucio Solano

nos referimos. Así mismo, hablando de forma general, sucede en mercados físicos, pero en los dos siempre el cliente debe observar el producto a comprar y en algunos casos hacer pruebas.

Por otro lado, la tecnología abre las puertas al desarrollo de emprendimientos, sean estos públicos y/o privados; la aparición de las aplicaciones móviles, del comercio electrónico hace que lo más insólito sea comercializado. Pero en realidad esto va más allá, se trata de analizar las dimensiones de la tecnología del comercio electrónico como es: usabilidad, alcance global, estándares universales, interactividad, densidad de la información, personalización y tecnología social (Laudon \& Traver , 2010).

Mejorar la distribución de los productos será la prioridad para cualquier comercio, hoy en día con la ayuda de la Web eso es posible por sus características y su naturaleza, el espacio que ha ganado durante los últimos años las tecnologías de la información y comunicación (TIC) ha modificado a la sociedad a la cultura y a la economía (Oliván 2016). El alcance que tiene dichos recursos que, un servicio sea más óptimo y eficaz en cuanto a promoción y venta y más efectivo y dinámico en la transacción comercial y en el servicio para los sujetos que intervienen.

Con esta referencia y vinculándole con la necesidad que tiene el MAGAP CHIMBORAZO, sería útil analizar la factibilidad de nuevas vías de comercialización basadas en las TICs, y mejorar los procesos de comercialización de los productos que ofertan las organizaciones vinculadas a la institución. Así no se limita a un día de comercio esporádico, permitiendo exhibir constantemente los productos deseados. Además, se puede lograr acortar brechas de intermediarios, haciendo que pequeños y medianos productores se organicen y utilicen este medio tecnológico; y en un futuro muy cercano, por qué no imaginar que esto sea replicado en todas las zonas que administra el MAGAP. Así mismo, Perozo Martín \& Chirinos Martínez (2019), comentan la importancia de generar políticas que permitan promover una gobernanza efectiva en los productores, por cuanto esto aporta a la soberanía alimentaria del país, por otro lado, 
lustitia Socialis. Revista Arbitrada de Ciencias Jurídicas.

Año IV. Vol. IV. N6. Enero - Junio 2019

Hecho el depósito de Ley: FA2016000064

ISSN: 2542-3371

FUNDACIÓN KOINONIA (F.K). Santa Ana de Coro, Venezuela

Alfredo Mauricio Astudillo Mamarandl; Mayra Gissela Lucio Solano

Colina (2018), esto es posible cuando se tiene apoyo en las TIC como recurso que permite mediar en la calidad del servicio prestado.

\section{DESARROLLO}

\section{Factores que justifican el funcionamiento legal del MAGAP}

"El Ecuador se encuentra entre los países que poseen una capacidad de producción de alimentos por encima de la creciente demanda de su población" (MAGAP, 2016), pero esto contrasta con la vulnerabilidad dentro del ámbito productivo y ecológico que sufren los campos agropecuarios en el país. Por tal motivo dentro del contexto ecuatoriano y como toda institución gubernamental, el Ministerio de Agricultura, Ganadería, Acuacultura y Pesca se alinea a la Constitución de la República, las políticas públicas de estrategias nacionales como: La Erradicación de la Pobreza (2014), Plan Nacional de Desarrollo 2017 - 2021 y en los postulados de la Agenda Productiva Sectorial, la cual busca fortalecer la presencia de los pequeños y medianos productores agrícolas, en áreas como cadenas productivas y líneas de comercialización.

Además, en un análisis en cuanto al lineamiento legal y políticas del MAGAP a nivel de la legislación ecuatoriana se revisó la política agraria en el marco actual del sistema de política pública, las cuales integran el marco estratégico de la institución en estudio, MAGAP en donde se identifican edictos que ayuden a justificar de mejor manera la propuesta a desarrollar, así tenemos:

- Según la Constitución de la República vigente en su artículo 281, literal 10, manifiesta "Fortalecer el desarrollo de organizaciones y redes de productores y de consumidores, así como la de comercialización y distribución de alimentos que promueva la equidad entre espacios rurales y urbanos". Así como también el artículo 304, literal 1, hace mención a "Desarrollar, fortalecer y dinamizar los mercados internos a partir del objetivo estratégico establecido en el Plan Nacional de Desarrollo."

- En cuanto al Plan Nacional de Desarrollo, dentro de su estructura general y enmarcados en sus ejes, manifiestan: Eje 2 - Economía al servicio de la sociedad, Objetivo 4 
lustitia Socialis. Revista Arbitrada de Ciencias Jurídicas.

Año IV. Vol. IV. Nº6. Enero - Junio 2019

Hecho el depósito de Ley: FA2016000064

ISSN: 2542-3371

FUNDACIÓN KOINONIA (F.K). Santa Ana de Coro, Venezuela

Alfredo Mauricio Astudillo Mamarandl; Mayra Gissela Lucio Solano

(Consolidar la sostenibilidad del sistema económico social y solidario...), en su política 4.7 hace mención a la inversión productiva privada en sus diversos esquemas, e incita a la asociatividad y alianzas público-privadas. Mientas que el objetivo 5 (Impulsar la Productividad y Competitividad para el Crecimiento Económico Sustentable...) en sus políticas $5.1,5.3,5.4,5.5$ y 5.8, hace referencia al incentivo dentro sector productivo, promoviendo la transferencia tecnológica, la innovación y el emprendimiento en base a las necesidades sociales. Todo esto alineado en la competitividad y calidad de productos primarios y el desarrollo de la industria agrícola para el fortalecimiento de la provisión de bienes y servicios.

Y en el Objetivo 6 (Desarrollar las Capacidades Productivas y del Entorno para Lograr la Soberanía Alimentaria y el Desarrollo Rural Integral), en su política 6.2 hace alusión al trabajo digno, al fortalecimiento y apoyo del estado impulsando el emprendimiento, al acceso del mercado y a la asociatividad social productiva para garantizar precios justos y control de contrabando.

- El Catálogo de Políticas del Consejo Sectorial de la producción, dentro de su eje COMPETITIVIDAD SISTÉMICA; Problemática 16: Insuficiente infraestructura de almacenamiento, acopio, vialidad, accesibilidad, conectividad, logística, movilidad. El cual promulga como Política Sectorial MAGAP: "Facilitar a los productores del sector agropecuario, acuícola y pesquero el acceso a los servicios de acopio y almacenamiento de la producción para garantizar condiciones justas de comercialización".

- La Agenda para la Transformación Productiva (2010), al establecer sus políticas, menciona una muy importante y relacionada al tema de investigación: Política de Comercialización que impulse procesos eficientes de: almacenamiento y distribución, precios (eliminación de intermediación ineficiente), formalización y acceso al mercado, agroindustria y comercio exterior.

Dentro de las leyes y políticas que rige el estado, existe criterios de coincidencia los cuales son: fortalecer, articular las organizaciones y redes de pequeños y medianos productores agrícolas, para promover una igualdad de condiciones en el mercado, así 
como también la eliminación prolongada de intermediarios garantizando escenarios de comercialización más justos.

\section{Ejes estratégicos del MAGAP}

El manejo y conservación de los recursos naturales (MAGAP, s.f.), es el objetivo fundamental de la institución, el cual se encuentra en estrecha relación con los 12 ejes estratégicos del estado para el sector agropecuario. Aquí llama la atención una en particular, Desarrollo de la Agroindustria, Mercados y Sistemas de Comercialización Internos y Externos.

Por otro lado, la visión institucional, declara: "Para el 2020 el Ministerio de Agricultura, Ganadería, Acuacultura y Pesca, contará con un modelo de gestión de calidad basado en sistemas de información y comunicación. En teoría posibilitan la producción de bienes y servicios que garanticen la seguridad alimentaria del país; además, el crecimiento y desarrollo equitativo dentro del área de concesión, generando valor agregado con rentabilidad económica, equidad social, sostenibilidad ambiental e identidad cultural”. Así consta en su página WEB oficial.

Estos 2 factores hacen mención al énfasis de la institución, por mejorar sus líneas de producción y comercialización, y el mismo está fundamentado en el uso de las nuevas tecnologías. Otro punto a favor para la construcción de una nueva línea de mercadeo de los productos auspiciados por el MAGAP.

\section{Programas y servicios}

EI MAGAP, a nivel nacional ejecuta programas y servicios que se aplica en las 9 zonas, a niveles administrativos de planificación, trazado por la SEMPLADES, el cual según dicha entidad ayuda a identificar las necesidades y soluciones en ámbitos de servicio público.

Dentro de los programas y servicios ejecutados a nivel nacional por el MAGAP, se encuentra el Programa de Desarrollo Rural, con su servicio de Redes Comerciales, el 
cual se tomó como punto de partida para analizar su contenido y relacionarlo con el tema y objetivos de la investigación. Se llegó a determinar su importancia dentro del desarrollo agrario ecuatoriano, al gestionar e impulsar estrategias de comercialización de las organizaciones campesinas adheridas a la institución gubernamental.

Sin duda alguna el Programa de Desarrollo Rural y su servicio de redes comerciales, busca generar un valor agregado de los productos campesinos y promueve el consumo social con responsabilidad ambiental. Pero algo que llama la atención cuando se habla de la gestión de circuitos alternativos solo se toma en cuenta la ejecución de ferias, registro de organizaciones campesinas y la zonificación de la comercialización (Redes Comerciales, MAGAP, 2017).

Un ejemplo concreto de lo antes mencionado ocurre en la cuidad de Riobamba, el MAGAP trabaja con 150 personas aproximadamente, asociadas en organizaciones de pequeña y mediana producción agrícolas, sus métodos de comercialización se limitan a ferias esporádicas que no exceden de 2 veces por semana en lugares como el Parque Guayaquil, Mercado Mayorista, Plaza Alfaro de la Ciudad de Riobamba.

\section{METODOLOGÍA}

Se genera dos campos de estudio por un lado está el factor legal y por el otro el estudio económico, para los dos casos se realiza un análisis descriptico, transversal y no experimental, ya que se analiza al contexto tal como se presente y se sugiere ciertas directrices dentro de cada aspecto para mejorar y proponer un proyecto factible de participación gubernamental.

\section{Evaluación Legal}

Para determinar los elementos legales se aplicó método cualitativo, bajo la técnica de la entrevista a expertos en diferentes áreas de conocimiento. El objetivo de este punto es determinar las barreras de entrada y salida debidamente sustentadas en ámbitos legales, 
lustitia Socialis. Revista Arbitrada de Ciencias Jurídicas.

Año IV. Vol. IV. N6. Enero - Junio 2019

Hecho el depósito de Ley: FA2016000064

ISSN: 2542-3371

FUNDACIÓN KOINONIA (F.K). Santa Ana de Coro, Venezuela

Alfredo Mauricio Astudillo Mamarandl; Mayra Gissela Lucio Solano

tributarios y de recaudación tributaria que debe tener un e-commerce en el entorno ecuatoriano.

Para el desarrollo de la misma se tomó en cuenta varios perfiles dentro del ámbito público privado, profesionales que ayuden a dilucidar problemas planteados en forma de preguntas semi-estructuradas. Aquí no tiene que ver directamente si la persona posee experiencia dentro del campo del comercio electrónico como usuario, al contrario, lo que se pretende es asimilar los conocimientos en diferentes áreas legales y saber cuáles se pueden considerar a la hora de lanzar el e-commerce. Cabe indicar que cada entrevistado tuvo un período de revisión y aceptación del cuestionario, en la cual se abordó el tema central que nos compete en la investigación, como es el e-commerce.

\section{Evaluación Financiera}

Dentro de este ámbito se debe tomar en cuanta algo muy importante. El proyecto no busca lucro, sino un desarrollo comercial, social y sostenible para pequeños productores agrícolas, por lo cual, se toma como punto de partida la metodología de Costo Beneficio (CB) propuesta y ejemplificada por el centro de "Estudios Técnicos Inc - ETI" " (Castañer, 2014). Se pretende llegar a cuantificar al máximo posible, los costos y beneficios (ingresos) en términos monetarios, con el fin de identificar si el costo de la solución sobrepasa al costo del problema. Cada análisis debe tener una secuencia y procedimiento a seguir es así que:

El primer paso fue estimar datos provenientes de las evaluaciones: a nivel legal, técnico y comercial, tomando los costos individuales y generales (subsidiados por el ente intermediario que es el MAGAP_Chimborazo). Al desarrollar cada ítem se detectó la necesidad de profesionales con perfiles adecuados en cada una de ellas. Para monetizar el desarrollo del e-commerce se tomó en cuenta a los profesionales que van a intervenir en la tarea con remuneraciones como servidores públicos. Y para el caso de estudió se tomó en cuenta los Grupos Ocupacionales que se rigen bajo la Resolución N MRL - 2012 - 0021 del Ministerio de Relaciones Laborales (Minsterio de Trabajo, 2012); el cual detalla 
los valores de la escala de remuneraciones mensuales unificadas. Estos datos están debidamente verificados y alineados en tiempo y espacio con los profesionales que cuenta la entidad pública para el desarrollo del proyecto.

El segundo paso, fue estimar los ingresos generados por las ventas de los productos agrícolas por parte de las organizaciones participantes en el proyecto. Esto salió en base a la proyección de ventas realizado anteriormente en el análisis del mercado, con un incremento del $20 \%$ por año.

El tercer paso, fuer proyectar un cash flow en base a los costos e ingresos del proyecto; todo este cúmulo de datos fueron proyectados a 5 años. Por sugerencias del departamento de comercialización del MAGAP- Riobamba y por política interna del departamento legal de la institución, los "Convenios Marcos" se firman con un mínimo de 2 años a un máximo de 5 años. Obtenido estos datos fue posible encontrar la Razón Beneficio Costo, el cual para ser admitida como positiva debe ser mayor que 1, siempre y cuando los beneficios superen los costes e incluso se consideraría una igualdad en el caso de ser necesario ya que el proyecto es con visión social.

Como último paso, luego de hallar la razón Costo-beneficio, se realizó un análisis comparativo en donde se puso de manifiesto la proyección de costos de la producción de las canastas solidarias. El objetivo de esta acción es denotar el incremento en los costos al no existir auspicio. Además, se pudo evidenciar los costos anuales y semestrales subsidiados por el MAGAP por cada integrante de las organizaciones participantes.

\section{RESULTADOS Y DISCUSIÓN}

\section{Evaluación Legal}

Desde una perspectiva general, en lo expuesto por los especialistas en áreas legales, tributarias y de recaudación municipal, manifiestan que se debe tener en cuenta la constitución de la empresa, cuáles son sus objetivos y para qué se crea (de forma lícita). El tema se abordó de una forma general, la intención de la entrevista es conocer desde 
lustitia Socialis. Revista Arbitrada de Ciencias Jurídicas.

Año IV. Vol. IV. N6. Enero - Junio 2019

Hecho el depósito de Ley: FA2016000064

ISSN: 2542-3371

FUNDACIÓN KOINONIA (F.K). Santa Ana de Coro, Venezuela

Alfredo Mauricio Astudillo Mamarandl; Mayra Gissela Lucio Solano

varios agentes de control, lo que necesita un negocio de características digitales, es así que:

- Desde el ámbito de la recaudación municipal, no existen normas, que regulen el comercio electrónico, en cuanto a las barreras de entrada y salida. Se toma en cuenta los permisos respectivos de funcionamiento, como uso de suelo, predio o contratos de arrendamiento, permisos sanitarios, pago a los bomberos, entre otro, siempre y cuando el negocio virtual necesite de una infraestructura física para su funcionamiento. Si ese es el caso, existen los trámites regulares para la apertura del mismo, en el caso de no requerir un espacio físico como tienda o bodega no requiere de dichos trámites ni pago de impuesto alguno.

- Desde el ámbito tributario, el comercio electrónico como el negocio habitual tiene deberes y derechos, es así que hasta el momento no existe dentro del ámbito tributario una ley o reglamento que solo regule este tipo de negocios. Además, el contribuyente debe cumplir sus deberes normales en cuanto a pago de impuestos, registrar sus ingresos, realizar retenciones (si es el caso), declarar al SRI y pagar sus obligaciones tributarias en los periodos adecuados. La evasión de impuestos en negocios de esas características es muy habitual ya que no son fáciles de detectar Las barreras de entrada o de salida que se consideraría es el registro único de contribúyete (RUC). En cuanto al cobro de impuesto y tramitología a este tipo de negocios, son los mismos que se efectúa a un comercio normal.

- Desde el ámbito de la jurisprudencia, mantiene que el comercio electrónico está regido por la; i) Ley de Comercio Electrónico, firma y mensajes de datos, ii) Reglamento de la Ley del Comercio Electrónico y iii) Reglamento para Acreditación de Servicios de Comercio Electrónico. Los cuales fungen como leyes que acogen a este sistema de relación económica en línea, y funcionan como agentes reguladores. Mucho tienen que ver con el uso de contenidos, protección de datos y básicamente estas leyes están ligadas a la Ley Orgánica de Defensa del Consumidor. En cuanto a las barreras de entrada y salida se considera la accesibilidad de la información, antes, durante y después de una compra, la responsabilidad de los contenidos, elementos de infraestructura de firma electrónica (siempre y cuando lo requiera), la protección de datos (manifestada en la 
recopilación, y uso de los mismos y solo con autorización del titular). En el cobro de impuestos como tal no existe ningún decreto, ya que el comercio electrónico es considerado como un sistema de relación económica y por tanto debe alinearse a la ley vigente del comercio normal y deberá cumplir con los requisitos que le imponga los organismos de control nacionales y locales en ámbitos fiscales y legales.

Como la entrevista se realizó de una forma semielaborada, el experto mencionó que se debe tener en cuenta, que la ley está dada para personas naturales, jurídicas y entidades estatales que realicen un tipo de comercio o apoyen al mismo dentro de sus competencias, por lo que se le planteó una consulta "existe en el País alguna ley, reglamento que impida que una institución pública impulse un negocio virtual". A eso contestó: dentro del ámbito público, cualquiera que fuese su naturaleza no lo puede hacer de forma directa, ya que esas actividades le competen al SERCOP (Servicio Nacional de Contratación Pública), "pero puede funcionar como intermediario" allí es distinto, todo estamento público debe servir a la ciudadanía para mejorar procesos y buscar el desarrollo de los más vulnerables.

Debe entenderse que cuando se trabaja como intermediario esto debe ser sin fines de lucro y que debe estar amparado en un Convenio Marco entre las 2 partes. Documento donde se especifique la labor de cada una, los compromisos, deberes y derechos de las partes, así como sanciones, tiempo de duración del convenio y sus firmas de respaldo por las autoridades competentes. Es así que se presenta a continuación el esquema legal que debe cumplir el MAGAP - Chimborazo ante la propuesta del e-commerce: 
lustitia Socialis. Revista Arbitrada de Ciencias Jurídicas.

Año IV. Vol. IV. N6. Enero - Junio 2019

Hecho el depósito de Ley: FA2016000064

ISSN: 2542-3371

FUNDACIÓN KOINONIA (F.K). Santa Ana de Coro, Venezuela

Alfredo Mauricio Astudillo Mamarandl; Mayra Gissela Lucio Solano

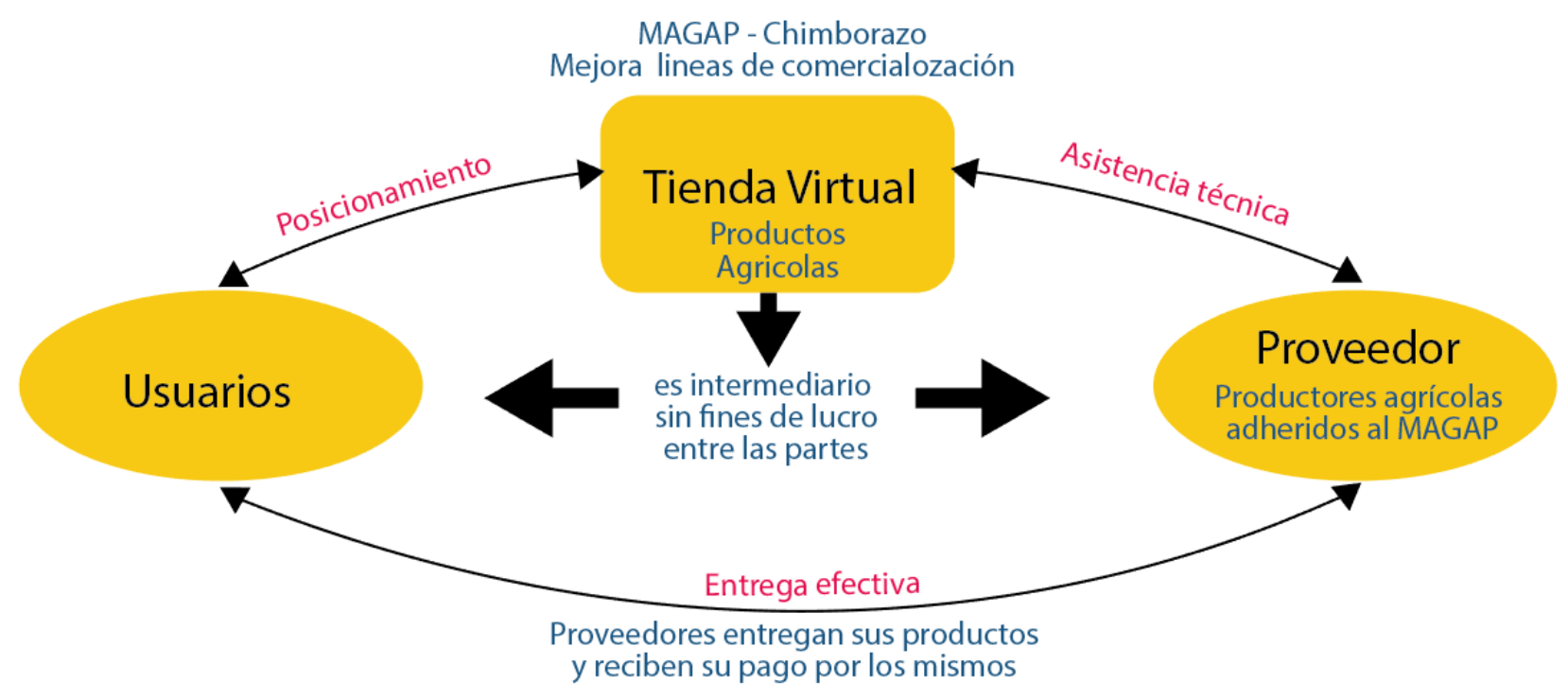

Figura 1. Esquema legal del modelo de negocio

Fuente: Esquema obtenido del estudio legal

\section{Evaluación Financiera}

Al desarrollar la evaluación financiera se determinó que los costos están en base a la categoría de Operación - Mantenimiento y se los toma como gastos corrientes por parte de institución gubernamental. Los beneficios están en base a los ingresos generados por el volumen de ventas, y se estima un incremento anual del $20 \%$ sobre los costos de producción a partir del segundo año del proyecto. Todo esto enmarcado en un canal paralelo de comprar y vender productos agrícolas.

Como primer paso se generó un cronograma valorado de los costos de implementación del sitio web, tomando en cuenta los gastos de: constitución, diseño - desarrollo e instalación y de comercialización, en el primer año al implementar el e-commerce, debería tener un desembolso inicial de VEINTIOCHO MIL OCHENTA Y UN DÓLARES (28,081.00 Dólares) por parte de la institución gubernamental auspiciante, para poder proyectar dichos gastos fue necesario depurar varios costos, ya que algunos rubros no se repiten en los años de duración del mismo, esto dio paso a estimar los gastos y los ingresos don mayor claridad y exactitud. 
lustitia Socialis. Revista Arbitrada de Ciencias Jurídicas.

Año IV. Vol. IV. Nº. Enero - Junio 2019

Hecho el depósito de Ley: FA2016000064

ISSN: 2542-3371

FUNDACIÓN KOINONIA (F.K). Santa Ana de Coro, Venezuela

Alfredo Mauricio Astudillo Mamarandl; Mayra Gissela Lucio Solano

Gastos proyectados. - En virtud del cumplimiento de las normativas del "Convenio Marco" se hace una estimación para 5 años, con un desembolso total de CIENTO VEINTICINCO MIL NOVECIENTOS NOVENTA Y OCHO DÓLARES (125,998.00 dólares), por parte de la institución gubernamental, entendiendo que es un gasto de operación y mantenimiento (Gasto Corriente), ya que la institución posee entre sus departamentos todo el equipo y profesionalismo para desarrollar este tipo de proyectos.

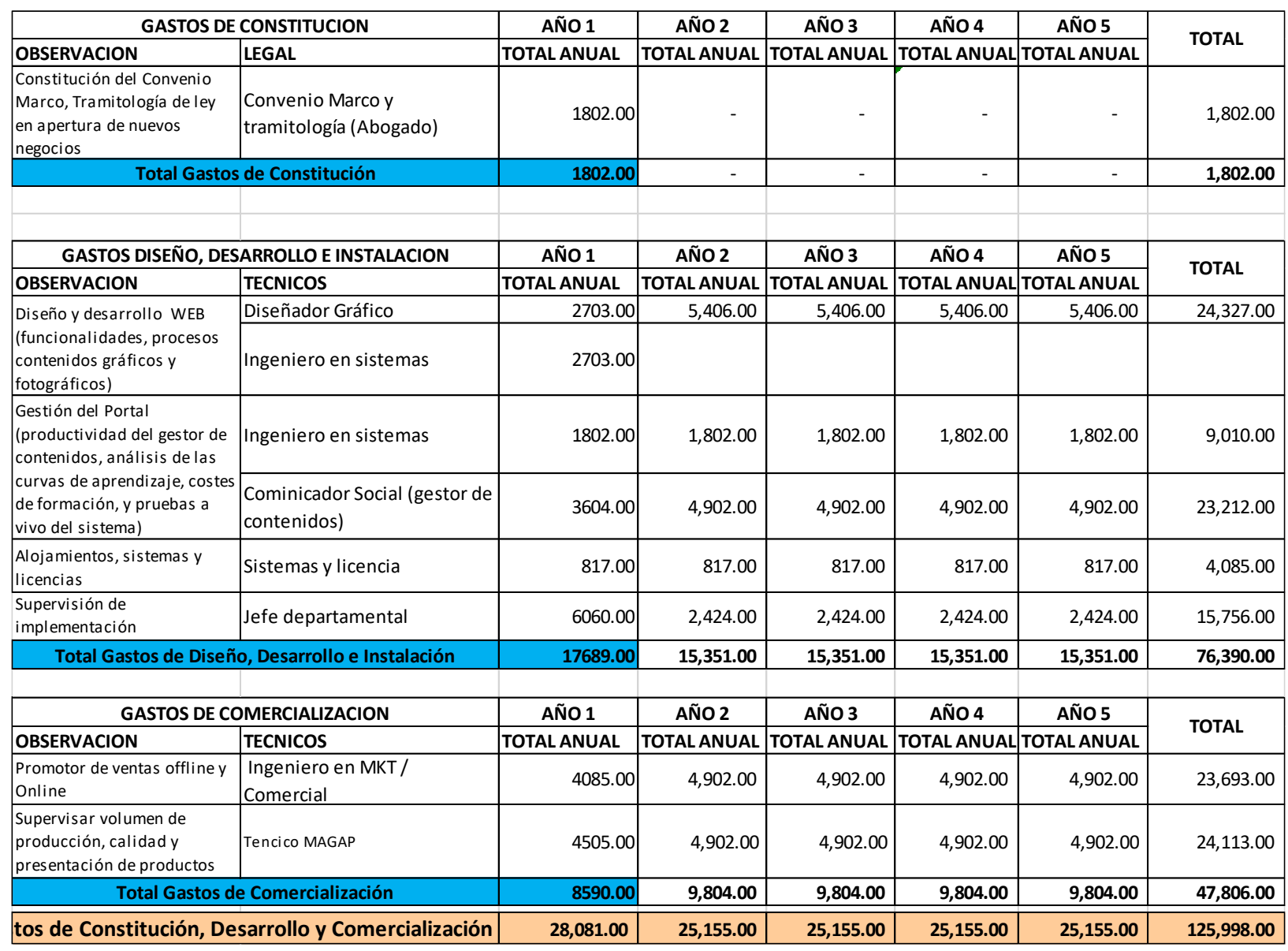

Figura 2. Gastos corrientes de tendrá que afrontar la empresa auspiciadora

Fuente: Datos obtenidos del estudio económico

Ingreso proyectado. - estos datos fueron facilitados por el departamento de comercialización del MAGAP-Chimborazo el punto de partida para la proyección de los 
lustitia Socialis. Revista Arbitrada de Ciencias Jurídicas.

Año IV. Vol. IV. №6. Enero - Junio 2019

Hecho el depósito de Ley: FA2016000064

ISSN: 2542-3371

FUNDACIÓN KOINONIA (F.K). Santa Ana de Coro, Venezuela

Alfredo Mauricio Astudillo Mamarandl; Mayra Gissela Lucio Solano

ingresos está en base al máximo de producción y venta de las organizaciones participantes en el proyecto.

\begin{tabular}{|c|c|c|c|c|c|c|}
\hline \begin{tabular}{|l} 
PRODUCCION Y VENTA \\
\end{tabular} & AÑO 1 & AÑO 2 & AÑO 3 & AÑO 4 & AÑO 5 & \multirow{2}{*}{ TOTAL } \\
\hline PRODUCCION DE CANASTAS & TOTAL ANUAL & TOTAL ANUAL & TOTAL ANUAL & TOTAL ANUAL & TOTAL ANUAL & \\
\hline Costo Total & $142,360.80$ & $170,832.96$ & $204,999.55$ & $245,999.46$ & $295,199.35$ & 2.13 \\
\hline Utilidad Ganada & $20,832.60$ & 24,9 & $29,998.94$ & $35,998.73$ & $43,198.48$ & $155,027.88$ \\
\hline Total Ingresos x Ventas Netas & $163,200.00$ & $195,832.08$ & $234,998.50$ & $281,998.20$ & $338,397.83$ & $1,214,426.61$ \\
\hline
\end{tabular}

Figura 3. Proyección en base al máximo de producción y ventas con un incremento anual del $20 \%$ anual

Fuente: Datos obtenidos del estudio económico

Análisis Costo Beneficio- Para identificar los beneficios netos, es necesario realizar un flujo de caja con las estimaciones realizadas entre los costos y los ingresos del proyecto:

\begin{tabular}{|c|c|c|c|c|c|c|}
\hline CASH FLOW & AÑO 1 & AÑO 2 & AÑO 3 & AÑO 4 & AÑO 5 & TOTAL \\
\hline Costos del Proyecto & $28,081.00$ & $25,155.00$ & $25,155.00$ & $25,155.00$ & $25,155.00$ & $128,701.00$ \\
\hline Beneficios o Utilidades & $20,832.60$ & $24,999.12$ & $29,998.94$ & $35,998.73$ & $43,198.48$ & $155,027.88$ \\
\hline CASH FLOW NETO & $7,248.40$ & 155.88 & $4,843.94$ & $10,843.73$ & $18,043.48$ & $26,326.88$ \\
\hline CASH FLOW NETO PORCENTUAL & $-74.19 \%$ & $-99.38 \%$ & $16.15 \%$ & $30.12 \%$ & $41.77 \%$ & $16.98 \%$ \\
\hline
\end{tabular}

\begin{tabular}{|c|r|}
\hline$\sum$ Beneficio & $155,027.88$ \\
\hline$\Sigma$ Costos & $128,101.00$ \\
\hline B/C & 1.21 \\
\hline
\end{tabular}

Figura 4. Cash Flow de los costos de implementación y los ingresos estimados

Fuente: Datos obtenidos del estudio económico

A primera vista los ingresos son superiores a los egresos. En el año 3 se acentúa un porcentaje positivo de los beneficios frente a los costos del proyecto, de allí en adelante se nota un incremento sustancial para el e-commerce, llegando a obtener un $16.98 \%$ de beneficio frente a los costos del proyecto en la vigencia del convenio, entre las 2 partes participantes. Al extraer la razón, beneficio costo (1.21), y ésta al ser mayor que 1, da muestras que el proyecto planteado tiene una factibilidad dentro del aspecto financiero.

Análisis comparativo. - Hipotéticamente, si el proyecto fuera de carácter privado y la implementación del e-commerce naciera de la misma, es obvio el incremento en los 
lustitia Socialis. Revista Arbitrada de Ciencias Jurídicas.

Año IV. Vol. IV. N6. Enero - Junio 2019

Hecho el depósito de Ley: FA2016000064

ISSN: 2542-3371

FUNDACIÓN KOINONIA (F.K). Santa Ana de Coro, Venezuela

Alfredo Mauricio Astudillo Mamarandl; Mayra Gissela Lucio Solano

costos y precio de venta al público. Esto se justifica por que se hiso un análisis comparativo contable, partiendo de los costos de producción y se detectó lo siguiente:

\begin{tabular}{|c|c|c|c|}
\hline \multirow{2}{*}{ Nivel de Produccion } & \multicolumn{2}{|r|}{ ANUAL } & ANUAL \\
\hline & $\mathrm{AU}$ & ISPICIADO & SIN AUSPICIO \\
\hline Materia Prima & $\$$ & $96,000.00$ & $\$ 96,000.00$ \\
\hline Mano de Obra Directa & $\$$ & $12,960.00$ & $\$ 12,960.00$ \\
\hline CIF & $\$$ & $13,600.80$ & $\$ 13,600.80$ \\
\hline Gastos de Operación & $\$$ & $19,800.00$ & $\$ 47,880.96$ \\
\hline Gastos Administrativos & $\$$ & $7,440.00$ & $\$ 7,440.00$ \\
\hline Gastos Distribucion/Ventas & $\$$ & $12,360.00$ & $\$ 12,360.00$ \\
\hline Gastos de implementacion de la tienda virtual & $\$$ & - & $\$ 28,081.00$ \\
\hline Gastos Financiamiento & $\$$ & - & $\$$ \\
\hline Costo de Produccion & $\$$ & $122,560.80$ & $\$ 122,560.80$ \\
\hline Costos Total & $\$$ & $142,360.80$ & $\$ 170,441.76$ \\
\hline Costo Unitario & $\$$ & 14.83 & 17.75 \\
\hline Utilidad Ganada (14.64\%) & $\$$ & 2.17 & 2.60 \\
\hline Precio de Venta & $\$$ & 17.00 & 20.35 \\
\hline Ventas Anuales & & $163,200.00$ & $\$ 195,394.43$ \\
\hline NUMERO DE CANASTAS & & 9600 & 9600 \\
\hline GANANCIA ANUAL & $\$$ & $20,841.62$ & $\$ 24,952.67$ \\
\hline
\end{tabular}

Figura 5. Proyección de venta anual con y sin auspicio del MAGAP

Fuente: Datos obtenidos del estudio económico

Desde una perspectiva general se nota un incremento considerable y una ganancia significativa, pero cabe mencionar que, si se incrementa los costos de la canasta solidaria, será muy difícil competir con los mercados formales e informales de la localidad. Por otro lado, reducir el margen de utilidad sería un riesgo muy alto.

También es importante saber el nivel monetario de participación de la institución auspiciante, para lograr entender la importancia del proyecto y el beneficio que se tiene el mismo. Si se divide el total de los costos de implementación del e-commerce a cada uno de los socios integrantes en el proyecto (Figura 1/ columna Año 1), se hallará el monto subsidiado por parte del organismo gubernamental. 
lustitia Socialis. Revista Arbitrada de Ciencias Jurídicas.

Año IV. Vol. IV. N6. Enero - Junio 2019

Hecho el depósito de Ley: FA2016000064

ISSN: 2542-3371

FUNDACIÓN KOINONIA (F.K). Santa Ana de Coro, Venezuela

Alfredo Mauricio Astudillo Mamarandl; Mayra Gissela Lucio Solano

Tabla 1.

Costos subsidiados al implementar el e-commerce por el MAGAP - Chimborazo

\begin{tabular}{cccc}
\hline Inversión anual & Beneficiarios & $\begin{array}{c}\text { Costo subsidiado anual por } \\
\text { beneficiario }\end{array}$ & $\begin{array}{c}\text { Costo subsidiado } \\
\text { mensual por } \\
\text { beneficiario }\end{array}$ \\
\hline 28081.00 & 150 & 187.21 & 46.80 \\
\hline
\end{tabular}

Al tomar el gasto corriente del primer año de funcionamiento del sitio web, desde la creación hasta la puesta en vivo a los usuarios, se requiere un valor de VEINTIOCHO MIL OCHENTA Y UN DÓLARES $(\$ 28.081,00)$. Al ser dividido para los 150 integrantes que conforman la CORPOCOSACH y AGROECOLÓGICA, quienes trabajan actualmente con el MAGAP en las ferias Solidarias. El costo que auspicia la institución gubernamental antes mencionada es de CIENTO OCHENTA Y SIETE DÓLARES, CON 21/100 CENTAVOS (\$187.21) anual aproximadamente, y de CUARENTA Y SEIS DÓLARES CON 80/100 CENTAVOS (\$46.80), mensuales por beneficiario.

\section{CONCLUSIÓN}

Partiendo del análisis de resultados desde la parte legal, el e-commerce, no se debe cumplir con ningún requisito en principio, pero hay que tener en cuenta la protección de datos al ejercer una venta y cumplir con los mismos requisitos que una tienda física (condición legal básica). Además, tener presente que, al momento de adquirir bienes y servicios por medios electrónicos, la Ley de Comercio Electrónico, Firmas y Mensajes de Datos (Art. 50), manifiesta, que antes de efectuarse la transacción, el proveedor debe informar detalladamente sobre todos los requisitos, restricciones, condiciones que tiene dicho producto o servicio que pretende adquirir el consumidor o usuario.

En cuanto a que una entidad pública, caso concreto el MAGAP puede ser intermediario para mejorar la comercialización de productos agrícolas. Según las formas de 


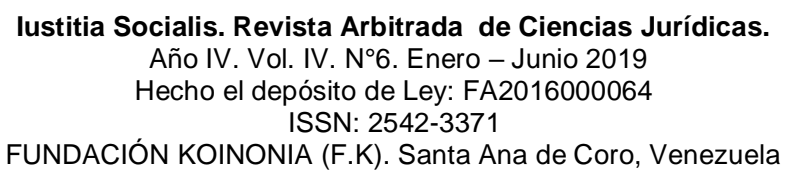

Alfredo Mauricio Astudillo Mamarandl; Mayra Gissela Lucio Solano

organización de la producción y su gestión, se manifiesta lo escrito en la constitución ecuatoriana en los artículos 319 y 320 que declara textualmente:

"Art. 319.- Se reconoce diversas formas de organización de la producción en la economía, entre otras las comunitarias, cooperativas, empresariales públicas o privadas, asociativas, familiares, domésticas, autónomas y mixtas. El estado promoverá las formas de producción que aseguren el buen vivir de la población y desincentivará aquellas..." y "Art. 320.- En las diversas formas de organización de los procesos de producción se estimulará una gestión participativa, transparente y eficiente. La producción, en cualquiera de sus formas, se sujetará a principios y normas de calidad, sostenibilidad, productividad sistemática, valoración del trabajo y eficiencia económica y social" (Constitución , 2008).

En conclusión, la constitución reconoce este tipo de organización, con sus debidas restricciones, porque el MAGAP solo podrá actuar como intermediario y ente de apoyo enmarcado en su legislación interna, con la creación del e-commerce. Y para que sea sustentado dentro de la legalidad se debe efectuar un convenio MARCO entre las partes interesadas, solo así podrá funcionar legalmente. Esto da luz verde para efectuar el proyecto $y$, por otro lado, los programas que ejecuta el MAGAP como son Desarrollo Rural con su proyecto Redes Comerciales.

Desde el ámbito económico al ser un proyecto que no busca lucro sino desarrollo social, en el ámbito económico se pudo evidenciar en primera instancia que el beneficio es mayor que los gastos. Así lo demostró la razón costo benéfico al ser mayor que 1. En segunda instancia se demostró que, si los costos de implementación del e-commerce fueran asumidos por las organizaciones, provocaría un incremento en el valor de los productos y su presentación como canastas, llegando a elevar su valor en más del 16\% de su costo real y por ende su penetración en el mercado riobambeño se dificultaría o sería poco dinámico.

Por otro lado, si equiparamos: al estado, a las organizaciones de pequeños y medianos productores agrícolas y la ciudadanía como actores que se verán impactados por un 
medio de comercialización virtual, se denotarán los beneficios para todas las partes. El estado por medio del MAGAP, desarrolla beneficios de gestión, dentro de lo humano, tecnológico, agrario e imagen institucional. Además de apostar a nuevas alternativas de desarrollo rural y sus servicios dentro de las redes de comercialización.

Las organizaciones agrícolas tendrán la oportunidad de interactuar directamente con el consumidor final y por ende de hacer crecer sus ventas, así como la producción, además, la ciudadanía puede acceder a un conjunto de beneficios económicos, de tiempo y de gasto, al disminuir la cadena de distribución de los productos agrícolas.

Aquí cabe la pregunta ¿es viable el proyecto en el aspecto financiero? Si se toma en cuenta que las ferias "YO PREFIERO" no tienen una infraestructura física propia o un espacio autorizado para desarrollar las actividades de comercio, su viabilidad es positiva. Se está dotando de un medio alternativo de comercio en donde se muestren los productos de forma constante. Además, hay un control de la variabilidad de productos, existen costos claros, con un piso y techo establecido de la demanda en función a la capacidad de producción.

Por último, al haber determinado el valor que la empresa gubernamental está aportando en términos económicos, hace pensar que es un proyecto que puede dar mucho dinamismo al comercio de productos agrícolas y así atrae otras asociaciones para que se unan a las actividades. Es claro que si se acrecienta el número de participantes no acrecentará los costos de la implementación del e-commerce y el mantenimiento de la misma.

\section{REFERENCIAS CONSULTADAS}

1. ETI, agencia peruana, especialista de estudios financiera, recuperado de: http://gis.jp.pr.gov/Externo_Econ/Talleres/PresentationCB_JP_ETI.pdf, muestra un ejemplo ilustrado de la Razón Beneficio Costo.

2. Castañer, J. (28 de 02 de 2014). Junta de Planificación / análisis de costo beneficio. Estudios Técnicos INC. Obtenido de http://gis.jp.pr.gov 


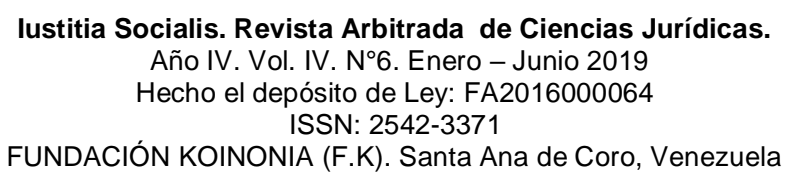

Alfredo Mauricio Astudillo Mamarandl; Mayra Gissela Lucio Solano

3. Constitución. (2008). Capitulo Sexto. Quito: Asamblea Constituyente.

4. Colina, M. (2018). Ambiente virtual de aprendizaje de contabilidad ii para las horas de trabajo independiente del programa nacional de formación en administración. Revista Arbitrada Interdisciplinaria Koinonía, 2(3), 92-108. Recuperado de http://fundacionkoinonia.com.ve/ojs/index.php/revistakoinonia/article/view/54/41

5. Laudon, K., \& Traver, C. (2010). E-commerce. México: PEARSON.

6. MAGAP. (2016). La política agropecuaria ecuatoriana al 2025. Obtenido de http://www.competencias.gob.ec/wp-content/uploads/2017/05/01PPP2016POLITICA01.pdf

7. Ministerio de Agricultura, Ganadería. (13 de 11 de 2017). Ministerio de Agricultura, Ganadería, Obtenido de Ministerio de Agricultura y Ganadería > El Ministerio > Valores / Misión / Visión: http://www.agricultura.gob.ec

8. Minsterio de Trabajo. (9 de 02 de 2012). Registro Oficial. Obtenido de http://www.trabajo.gob.ec

9. OLIVÁN, R. La cuarta Revolución Industrial, un relato desde el materialismo cultural. URBS: Revista de Estudios Urbanos y Ciencias Sociales, 2016, 6 (2): p. 101-111.

10. Perozo Martín, R., \& Chirinos Martínez, A. (2019). Incidencias de la Tecnología web 2.0 en el contexto de la gobernanza y la gobernabilidad. IUSTITIA SOCIALIS, 4(6), 90-116. doi:http://dx.doi.org/10.35381/racji.v4i6.291

11. Redes Comerciales, MAGAP. (06 de 12 de 2017). agricultura.gob.ec. Obtenido de Ministerio de Agricultura y Ganadería > Programas y Servicios > Redes Comerciales: http://www.agricultura.gob.ec/redes-comerciales/

\section{CONSULTED REFERENCE}

1. ETI, Peruvian agency, financial studies specialist, retrieved from: http://gis.jp.pr.gov/Externo_Econ/Talleres/PresentationCB_JP_ETI.pdf, shows an illustrated example of the Cost Benefit Reason. 
lustitia Socialis. Revista Arbitrada de Ciencias Jurídicas.

Año IV. Vol. IV. N6. Enero - Junio 2019

Hecho el depósito de Ley: FA2016000064

ISSN: 2542-3371

FUNDACIÓN KOINONIA (F.K). Santa Ana de Coro, Venezuela

Alfredo Mauricio Astudillo Mamarandl; Mayra Gissela Lucio Solano

2. Castañer, J. (28 of 02 of 2014). Planning Board / cost benefit analysis. Technical Studies INC. Retrieved from http://gis.jp.pr.gov

3. Constitution. (2008). Sixth chapter. Quito: Constituent Assembly.

4. Colina, M. (2018). Virtual accounting learning environment ii for working hours independent of the national administration training program. Interdisciplinary Arbitrated Review Koinonía, 2 (3), 92-108. Recovered from http://fundacionkoinonia.com.ve/ojs/index.php/revistakoinonia/article/view/54/4

5. Laudon, K., \& Traver, C. (2010). E-commerce Mexico: PEARSON.

6. MAGAP. (2016). Ecuadorian agricultural policy by 2025. Retrieved from http://www.competencias.gob.ec/wp-content/uploads/2017/05/01PPP2016POLITICA01.pdf

7. Ministry of Agriculture, Livestock. (13 of 11 of 2017). Ministry of Agriculture, Livestock, Obtained from the Ministry of Agriculture and Livestock> The Ministry> Values / Mission / Vision: http://www.agricultura.gob.ec

8. Ministry of Labor. (9 of 02 of 2012). Official register. Obtained from http://www.trabajo.gob.ec

9. OLIVÁN, R. The fourth Industrial Revolution, a story from cultural materialism. URBS: Journal of Urban Studies and Social Sciences, 2016, 6 (2): p. 101-111.

10. Perozo Martín, R., \& Chirinos Martinez, A. (2019). Incidents of Web 2.0 Technology in the context of governance and governance. IUSTITIA SOCIALIS, 4 (6), 90-116. doi: http: //dx.doi.org/10.35381/racji.v4i6.291

11. Commercial Networks, MAGAP. (06 of 12 of 2017). agriculture.gob.ec. Obtained from the Ministry of Agriculture and Livestock> Programs and Services> Commercial Networks: http://www.agricultura.gob.ec/redes-comerciales/

\footnotetext{
(C)2019 por los autores. Este artículo es de acceso abierto y distribuido según los términos y condiciones de la licencia Creative Commons Atribución-NoComercial-Compartirlgual 4.0 Internacional (CC BY-NC-SA 4.0) (https://creativecommons.org/licenses/by-nc-sa/4.0/).
} 